BNL-113650-2017-JA

\title{
Antifogging abilities of model nanotextures
}

\author{
Timothée Mouterde, Gaëlle Lehoucq, Stéphane Xavier, \\ Antonio Checco, Charles T. Black, Atikur Rahman, \\ Thierry Midavaine, Christophe Clanet \& David Quéré
}

Submitted to the Journal of Nature Materials

February 2017

Center for Functional Nanomaterials

Brookhaven National Laboratory

\author{
U.S. Department of Energy \\ USDOE Office of Science (SC), \\ Basic Energy Sciences (BES) (SC-22)
}

\footnotetext{
Notice: This manuscript has been authored by employees of Brookhaven Science Associates, LLC under Contract No. DE- SC0012704 with the U.S. Department of Energy. The publisher by accepting the manuscript for publication acknowledges that the United States Government retains a non-exclusive, paid-up, irrevocable, world-wide license to publish or reproduce the published form of this manuscript, or allow others to do so, for United States Government purposes.
} 


\section{DISCLAIMER}

This report was prepared as an account of work sponsored by an agency of the United States Government. Neither the United States Government nor any agency thereof, nor any of their employees, nor any of their contractors, subcontractors, or their employees, makes any warranty, express or implied, or assumes any legal liability or responsibility for the accuracy, completeness, or any third party's use or the results of such use of any information, apparatus, product, or process disclosed, or represents that its use would not infringe privately owned rights. Reference herein to any specific commercial product, process, or service by trade name, trademark, manufacturer, or otherwise, does not necessarily constitute or imply its endorsement, recommendation, or favoring by the United States Government or any agency thereof or its contractors or subcontractors. The views and opinions of authors expressed herein do not necessarily state or reflect those of the United States Government or any agency thereof. 
BNL-113650-2017-JA

\title{
Antifogging abilities of model nanotextures
}

\author{
Timothée Mouterde ${ }^{1,2}$, Gaëlle Lehoucq ${ }^{3}$, Stéphane Xavier ${ }^{3}$, \\ Antonio Checco ${ }^{4}$, Charles T. Black ${ }^{5}$, Atikur Rahman ${ }^{5}$, \\ Thierry Midavaine ${ }^{6}$, Christophe Clanet ${ }^{1,2} \&$ David Quéré ${ }^{1,2}$ \\ 1. Physique et Mécanique des Milieux Hétérogènes, UMR 7636 du CNRS, ESPCI, 75005 Paris, France. \\ 2. LadHyX, UMR 7646 du CNRS, École polytechnique, 91128 Palaiseau, France. \\ 3. Thales Research \& Technology, Route Départementale 128, 91767 Palaiseau, France. \\ 4. Condensed Matter Physics and Materials Science Department, Brookhaven National Laboratory, Upton, NY 11973, USA. \\ 5. Center for Functional Nanomaterials, Brookhaven National Laboratory, Upton, NY 11973, USA. \\ 6. Thales Optronique SAS, 2 avenue Gay-Lussac, 78990 Élancourt, France.
}

Nanometer-scale features with special shapes impart a broad spectrum of unique properties to the surface of insects. These properties are essential for the animal's survival, and include the low light reflectance of moth eyes, the oil repellency of springtail carapaces and the ultra-adhesive nature of palmtree bugs. Antireflective mosquito eyes and cicada wings are also known to exhibit some antifogging and self-cleaning properties. In all cases, the combination of small feature size and optimal shape provides exceptional surface properties. In this work, we investigate the underlying antifogging mechanism in model materials designed to mimic natural systems, and explain the importance of the texture's feature size and shape. While exposure to fog strongly compromises the water-repellency of hydrophobic structures, this failure can be minimized by scaling the texture down to nanosize. This undesired effect even becomes non-measurable if the hydrophobic surface consists of nanocones, which generate antifogging efficiency close to unity and water departure of droplets smaller than $2 \mu \mathrm{m}$. 


\section{Introduction}

Although textured hydrophobic materials show spectacular water-repellency, which causes millimetre-size drops to bounce off such surfaces ${ }^{1-2}$, they generally get wet when exposed to fogs or to humid atmosphere ${ }^{3-9}$. Droplets of a size comparable to that of the surface features can nucleate and grow within - rather than atop - the texture, and this so-called Wenzel state destroys superhydrophobicity ${ }^{6-9}$. Water strongly pinned in this way remains stuck as dew accumulates, resulting in hydrophilic-like behaviour. A large drop contacting this infused solid will also be pinned, owing to the multiple bridges provided by the subjacent wet patches. In humid air, water drops of any size between mist and rain get captured on the previously repellent material, even when it is tilted ${ }^{3-5}$.

These considerations suggest that more effective anti-fogging might result from scaling the texture sizes to the submicrometer range $\mathrm{e}^{10-11}$, which has other practical benefits such as rendering the coating robust against pressure ${ }^{12}$, or enhancing optical transparency ${ }^{13}$ and heat transfer $^{14-15}$. It has been reported that droplets of 10-100 $\mu \mathrm{m}$ condensing on nanotextures can remain mobile enough to allow an efficient transfer of surface energy gained in coalescence to kinetic energy, causing them to sometimes jump off the surface ${ }^{15-18}$. This property has been observed on solids with two-tier roughness ${ }^{19-21}$ or colloids ${ }^{22}$, and on cicadae wings covered with nanocones ${ }^{11}-$ thus potentially self-cleaned by fogs.

\section{Model nanotextures}

Inspired by these natural examples, we investigate how the anti-fogging properties of model patterned solids are impacted firstly by reducing the texture size, and secondly by changing the texture shape. A first series of substrates is uniformly decorated by pillars with accuratelycontrolled geometry, and long and dense enough to maintain Cassie configurations even for micrometric drops ${ }^{23-24}$. The pillars we designed have aspect ratios of 2-3 and spacing $p$ comparable to their height $h$. Scanning electron microscope (SEM) viewgraphs show these pillar arrays (Figure 1a). Sample A has the smallest structures, yet large enough to neglect wetting anomalies ${ }^{25}$ or structural effects in the liquid ${ }^{26}$. Posts have a radius $a=15 \mathrm{~nm}$ and a height $h=88 \mathrm{~nm}$, and they are disposed on an array of rhombuses with side $p=52 \mathrm{~nm}^{27}$. The roughness factor $r$, ratio of the total to apparent surface area of the solid, is $r_{A} \approx 4.5$. Textures B1, B2 and B3 are homothetic square lattices of posts with a pillar density around $10 \%$, an 
aspect ratio $h / 2 a=3$, and a roughness $r_{B} \approx 2.2$. The periodicity $p$ is $560 \mathrm{~nm}(\mathrm{~B} 1), 840 \mathrm{~nm}$ (B2) and $1120 \mathrm{~nm}$ (B3), respectively. Surfaces A and B are rendered hydrophobic by vapour deposition of $1 \mathrm{H}, 1 \mathrm{H}, 2 \mathrm{H}, 2 \mathrm{H}$-perfluorodecyltrichlorosilane. This treatment on flat silicon provides an advancing angle for water of $120^{\circ} \pm 2^{\circ}$.

Roughness enhances hydrophobicity; for textures B1-3, of constant density, we $\operatorname{expect~}^{28}$ and observe comparable advancing and receding water contact angles $\theta_{\mathrm{a}}=168^{\circ} \pm 2^{\circ}$ and $\theta_{\mathrm{r}}=143^{\circ}$ $\pm 3^{\circ}$. Sample A has similar wetting characteristics, with $\theta_{\mathrm{a}}=167^{\circ} \pm 2^{\circ}$ and $\theta_{\mathrm{r}}=140^{\circ} \pm 2^{\circ}$, despite its larger pillar density (which we discuss in the Supplementary Note 1). Anyway, the similarity in wettability allows us to compare materials with the same effective surface energy, but different structure size and periodicity.

\section{Condensation-induced adhesion}

In order to quantify the water-repellency of surfaces exposed to fogs, we examined the adhesion of drops having a temperature $T_{d}$ larger than that of the substrate, $T_{o}$ (Figure $1 \mathrm{~b}$ ). Water evaporates and recondenses in the textures, and the parameter $\Delta T=T_{d}-T_{o}$ allows us to continuously tune the atmosphere from relatively dry $(\Delta T=0)$ to highly foggy $(\Delta T>0)$. It has been reported that heated water often destroys superhydrophobicity ${ }^{8,29}$ : at large $\Delta T$, many water nuclei form and grow beneath the drop, which eventually sticks it to its substrate (Figure 1c).

We affix the centimetre-size samples to a brass block anchored at room temperature $\left(T_{o}=24 \pm\right.$ $1{ }^{\circ} \mathrm{C}$ ) and tilted by $\alpha=20^{\circ}$. Water at a controlled temperature $T_{d}=T_{o}+\Delta T$ is dispensed from a needle of diameter $d=210 \mu \mathrm{m}$ at a rate $Q=0.25 \mathrm{~mL} / \mathrm{min}$, so that millimetre-sized drops are inflated in a few seconds. We measure the drop mass $m$ as it detaches from the needle, that is, when its projected weight $m g \sin \alpha$ ( $g$ is the gravity acceleration) overcomes both the adhesion $F$ on the surface and the capillary force $\pi d \gamma$ of the needle, denoting $\gamma$ as the surface tension of water. Hence we deduce the adhesion force: $F=m g \sin \alpha-\pi d \gamma$. 


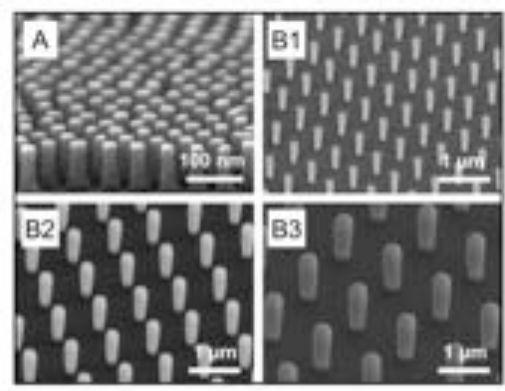

(a)

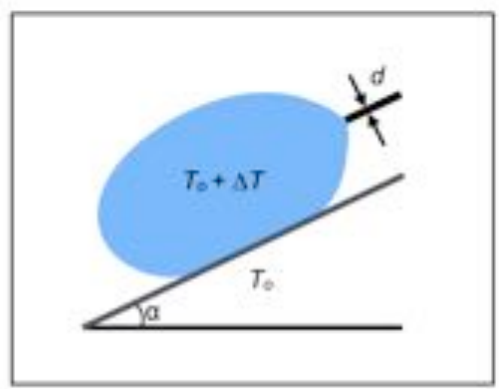

(b)

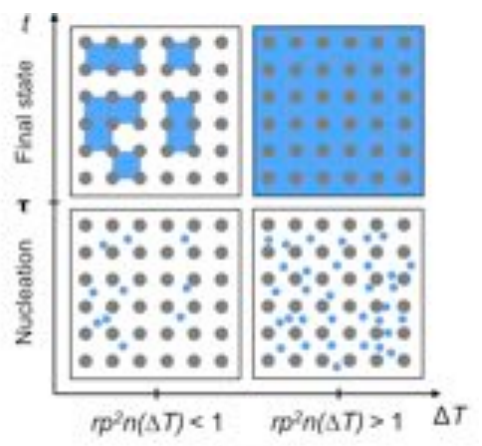

(c)

Figure 1. Hot drops on hydrophobic pillars. (a) Scanning electron microscope images of the pillar textures used in this study. On sample A, the pillars have a height $h=88 \mathrm{~nm}$, radius $a=15 \mathrm{~nm}$ and spacing $p=52 \mathrm{~nm}$. Samples B are homothetic, with pillars of aspect ratio 3 on a square lattice with spacing $p=560 \mathrm{~nm}$ (B1), 840 $\mathrm{nm}$ (B2) and $1120 \mathrm{~nm}$ (B3). (b) Experimental protocol: A needle of diameter $d$ dispenses water at temperature $T_{d}=T_{o}+\Delta T$ on hydrophobic textures at temperature $T_{o} \approx 24^{\circ} \mathrm{C}$. The samples are tilted by $\alpha=20^{\circ}$, and we measure the mass $m$ of the drop when it detaches from the needle. (c) Schematic of the condensation steps in a cold texture beneath a hot drop, as a function of $\Delta T$ (horizontal axis) and time (vertical axis). Grey and blue dots respectively show the lattice of pillars and the nuclei of water (of density $n$ ) condensing on the substrate. At small $\Delta T$, there is on average less than one nucleus per cell, which generates disconnected patches when cells are filled $(t>\tau)$. At large $\Delta T$, there is at least one nucleus per cell, so that water later invades all the structures.

Two factors contribute to $F$. First, pinning of water on pillars generates an intrinsic adhesion $F_{o}=F(\Delta T=0)$, as expressed by the contact angle hysteresis $\Delta \theta=\theta_{\mathrm{a}}-\theta_{\mathrm{r}}$ (constant for all samples). $F_{0}$ is assumed independent of temperature, owing to the modest variation of $\gamma$ as $T_{d}$ increases. $\mathrm{Yu}$ et al. indeed reported that water-repellency is maintained if both the substrate and water are heated, keeping $\Delta T=0^{29}$. Second, additional pinning will result from the nucleation and growth of droplets inside the texture for $\Delta T>0$ (Figure 1c). We assume the total adhesion $F$ is the sum of these two contributions, so that the condensation-induced adhesion $\Delta F=F(\Delta T)-F_{0}$ can simply be deduced from $m$ and $F_{0}$.

Figure 2a shows successive contours of inflating drops (black profiles), until detachment (red profile). Surface A ( $p=52 \mathrm{~nm})$ shows little change in the contour of detaching droplets with increasing $\Delta T$. In contrast, heating water dramatically amplifies adhesion on surface B2 ( $p=$ $840 \mathrm{~nm}$ ), where drops can become 10 times heavier without moving, even for $\Delta T$ as modest as $15{ }^{\circ} \mathrm{C}$ (Supplementary Movies 1-2). The receding angle in this case reduces significantly (while the advancing angle does not seem affected), which is indicative of stronger pinning.

The mass $m$ of detaching drops is found to be roughly independent of the injection rate $Q$ between 0.01 and $0.3 \mathrm{~mL} / \mathrm{min}$ (as reported in the Supplementary Note 2). At higher flow rates, 
inertia makes water come out of the needle as a jet, a regime that can also be tested. Figure $2 b$ shows jets dispensed at $Q=1 \mathrm{~mL} / \mathrm{min}$ and impacting samples A and B2 (Supplementary Movies 3-4). The results are fully consistent with observations in Figure 2a: both samples exhibit a similar behaviour for $\Delta T=0$ (the hallmark of hydrophobicity being here jet rebound) whereas repellency is destroyed on $\mathrm{B} 2$ and preserved on $\mathrm{A}$ for $\Delta T=15^{\circ} \mathrm{C}$ (or more). Hence repellency of hot water by sample A also holds under dynamic conditions (Supplementary Note 3). In addition, the reflection of hot jets on $\mathrm{A}$ is independent of the impact duration in the range 1-1000 s, as shown in Figure 2c.

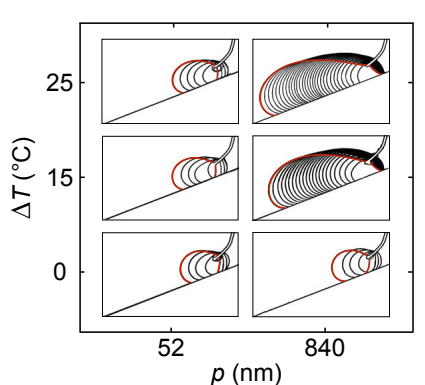

(a)

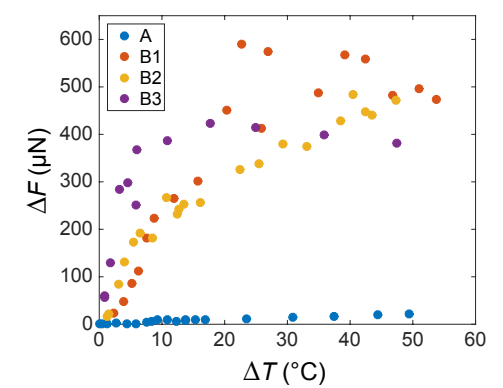

(d)

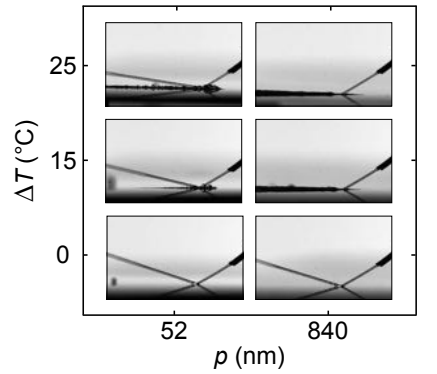

(b)

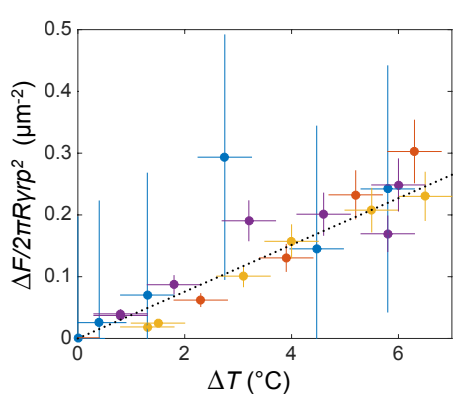

(e)

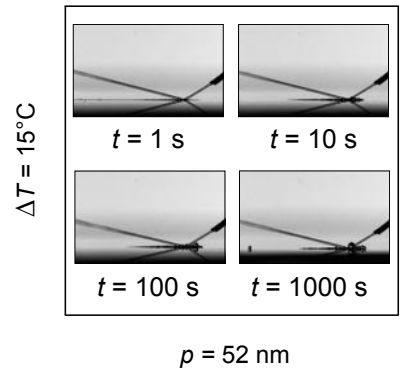

(c)

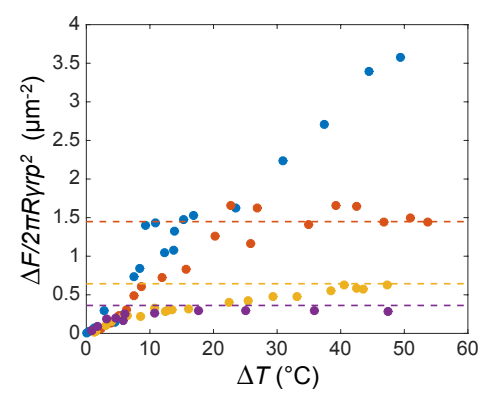

(f)

Figure 2. Adhesion of cold and hot water on materials A and B. (a) Successive profiles of water drops inflated either at the substrate temperature $(\Delta T=0)$, or at a larger temperature $\left(\Delta T=15\right.$ and $\left.25^{\circ} \mathrm{C}\right)$ on materials $\mathrm{A}(p=52$ $\mathrm{nm})$ and B2 $(p=840 \mathrm{~nm})$. Time interval between two contours is $0.75 \mathrm{~s}$, and inflating flux $Q$ is $0.25 \mathrm{~mL} / \mathrm{min}$. The red contour corresponds to the maximum mass $m$ immobilised on the solid. (b) At higher flow rate (here $Q=$ $1 \mathrm{~mL} / \mathrm{min}$ ), water comes out of the needle as a jet, whose behaviour on samples A and B2 is shown as a function of temperature $\Delta T$. (c) For sample $\mathrm{A}$, the rebound of a hot jet does not depend on the duration $t$ of impact. (d) Increment of adhesion force $\Delta F$ due to a temperature difference $\Delta T$ between water and its substrate for the surfaces of Figure 1a. (e-f) Adhesion force $\Delta F$ normalized by its maximum $2 \pi R \gamma$, and presented per unit area $r p^{2}$ of pillar cell, as a function of $\Delta T$. The colour code of the data points is the same as in (d). In (e), data at small $\Delta T$ collapse on the same curve (the dotted line is a guide for the eyes). In (f), data separate at larger $\Delta T$, and coloured dashed lines are the plateaus $1 / r p^{2}$ predicted by Eq. 1, for each B-substrate. The parameter $1 / r p^{2}=82 \mu \mathrm{m}^{-2}$ for surface $\mathrm{A}$ is out of the scale. 
The condensation-induced adhesion $\Delta F$ is plotted in Figure $2 \mathrm{~d}$ as a function of $\Delta T$ for $0<\Delta T<50^{\circ} \mathrm{C} . \Delta F$ increases with $\Delta T$ for all textures, although with quantitative differences between them. On the one hand, as observed in Figure 2a, adhesion on the smallest features hardly varies on sample $\mathrm{A}$ (at the scale of the plot), even for $\Delta T$ as large as $50^{\circ} \mathrm{C}$. On the other hand, adhesion on larger features (samples B1-3) markedly increases with $\Delta T$ and reaches a common plateau at $450 \pm 50 \mu \mathrm{N}$, a value about 10 times larger than the adhesion at room temperature $F_{o} \approx \pi R \gamma \Delta(\cos \theta) \approx 50 \pm 10 \mu \mathrm{N}$, where $R$ is the (millimetre-size) radius of the contact line at drop departure. In addition, the slope of $\Delta F$ at small $\Delta T$ increases with texture size and spacing, so that the plateau for larger textures is reached at smaller temperature differences.

We understand the reasons why smaller feature sizes resist the loss of superhydrophobicity using a straightforward model of condensation. As warm water contacts cold textures, we expect vapour to condense inside the texture voids. Once patches of water fill the voids, they provide strong bridges between the drop and the substrate. We characterize the first stage of condensation by the number $n$ of water nuclei per unit area. As sketched in Figure 1c, we divide the substrates (seen from the top) into elementary cells delimited by four pillars. The actual surface area of each cell is $r p^{2}$ so that the average number of nuclei per cell is $P=r p^{2} n$, which increases with $\Delta T$ as $n$ does. We successively distinguish the case $P<1$, for which wet cells remain disconnected, and $P>1$, for which all the cells under the drop are filled by water.

At small $\Delta T$ (bottom left in Figure 1c), an elementary cell contains on average less than one nucleus $\left(r p^{2} n<1\right)$. As time progresses, nuclei grow, fill the cells and contact the large drop resting on the pillars' top. Micrometric cells fill rapidly (with $\tau \sim 1-100 \mathrm{~ms}^{31}$ ), as detailed in the Supplementary Note 3. We assume that water stays enclosed within each cell, blocked by the outlying pillars (see the Supplementary Note 4). Then, as sketched in Figure 1c (top left), the breath figure eventually consists of a collection of water cells connected to the drop and increasing its adhesion. The corresponding force is obtained by multiplying $P$ by the perimeter $\pi R$ of the trailing edge of the drop, and by $2 \gamma$ (since two interfaces are generated when water leaves a filled cavity). Hence we find:

$$
\Delta F \approx 2 \pi R \gamma r p^{2} n
$$


This equation, derived in Supplementary Note 5, predicts that condensation-induced adhesion strongly depends on the structure size (via $p$ ) and aspect ratio (via $r$ ), and that it increases with $\Delta T$, as $n$ does. Eq. 1 can be tested, and we plot in Figure 2e-f the ratio $\Delta F / 2 \pi R \gamma\left(T_{d}\right) r p^{2}$ (in $\mu \mathrm{m}^{-2}$ ) as a function of $\Delta T$. We use the raw data of Figure $2 \mathrm{~d}$ and measure for each experiment the radius $R$ of the trailing contact line, found to be $\sim 1.2 \mathrm{~mm}$ for both low and high conditions of adhesion (see Supplementary Note 6). Two main regimes are observed. (i) At small $\Delta T$, all data collapse on a single curve (Figure 2e), in agreement with Eq. 1 that predicts $\Delta F / 2 \pi R \gamma r \lambda^{2}=n(\Delta T)$, the nucleation density on a hydrophobic material. We find that $n$ is typically $0.1-0.2 \mu \mathrm{m}^{-2}$, in agreement with the literature ${ }^{30}$, and that it increases with $\Delta T$ : larger temperature differences naturally favour nucleation. (ii) At large $\Delta T$ (Figure 2f), the data series separate from each other, and plateau at different values for samples B1-3. When the nucleation density reaches $n=1 / r p^{2}$, we expect at least one nucleus per cell (bottom right in Figure 1c), which later leads to the filling of all cells (top right in Figure 1c). At even larger $\Delta T, r p^{2} n$ exceeds 1 , but the final state remains the same. The corresponding saturation value $\Delta F_{\text {max }}$ is obtained by making $r p^{2} n=1$ in Eq. 1 , which yields:

$$
\Delta F_{\max }=2 \pi R \gamma
$$

Then the drop is in a Wenzel state induced by the substantial nucleation and growth of nuclei. $\Delta F_{\max }$ is the maximum of adhesion $F \approx \pi R \gamma \Delta(\cos \theta)$ reached for $\Delta(\cos \theta)=2$. A Wenzel advancing angle can remain large while a receding one, obtained as water detaches from trapped water, is minimum, which maximizes $\Delta(\cos \theta)$. In the representation adopted in Figure 2e-f, this limit implies a plateau $\Delta F_{\max } / 2 \pi R \gamma r p^{2}$, that is, $1 / r p^{2}$, the inverse cell area. Its known value marked in Figure $2 \mathrm{f}$ with dashed lines is found to be in excellent agreement with the measurements. We also understand why the plateau is not reached for sample A for which we have $1 / r p^{2} \approx 82 \mu \mathrm{m}^{-2}$, by far larger than the $\Delta F / 2 \pi R \gamma r p^{2}$ values. Hence we expect drops on very small textures to remain in the Cassie state in a much broader range of temperatures, which explains that the low adhesion reported in Figures $2 \mathrm{a}$ and $2 \mathrm{~d}$ results from the fine subdivision of water in fine features. 


\section{Feature shape effect}

While the texture size impacts a hydrophobic surface's ability to resist fogging, its shape can further enhance the effect. Here, we compare the behaviour of cylindrical and conical nanopillars undergoing water condensation. A fifth model material used in this study (Sample C, Figure 3a) is designed with a texture similar to that on the wings of the cicada Psaltoda claripennis (Figure 3b), for which antifogging properties were reported ${ }^{11}$.

(a)

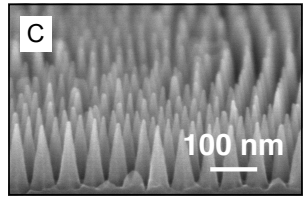

(b)

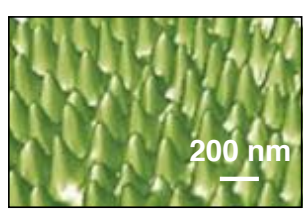

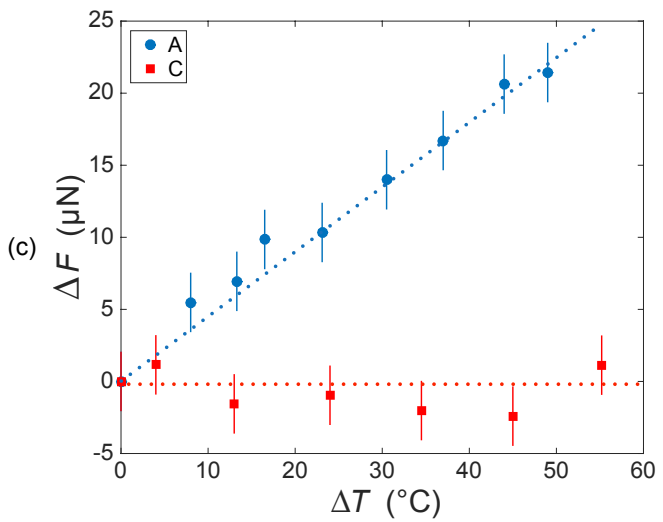

(d)

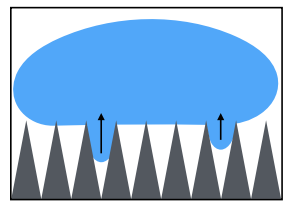

(e)

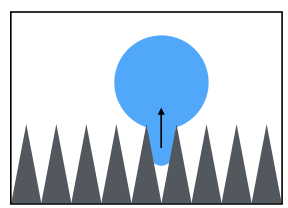

Figure 3. Comparison of the adhesion of hot drops on nanopillars and on nanocones. (a) SEM image of the nanocones (sample C) used in this study. They are $115 \mathrm{~nm}$-high and disposed on a lattice with spacing $p=52 \mathrm{~nm}$. (b) Nanotextures on cicada (Psaltoda claripennis) wings shown by atomic force microscopy (picture adapted from ref. 11). (c) Adhesion force $\Delta F$ due to condensation, as a function of temperature difference $\Delta T$ between water and substrate. We compare the adhesion on substrate A (blue circles, see also Figure $2 \mathrm{~b}$ ) to that on substrate C (red squares). (d) Sketch showing the effect of geometry: droplets beneath a large drop can be reabsorbed. (e) Similarly, small droplets condensing in cones can reconfigurate at the cone tops.

Surface $\mathrm{C}$ is covered by a dense array of nanocones with roughness $r_{C} \approx 4.2$ and treated by the same silane as previous samples. The conical structures with height $h=115 \mathrm{~nm}$ are created via the approach used for Sample A (Figure 1a), with slight changes to the etching conditions $^{27}$. The spacing (or base diameter) $p=52 \mathrm{~nm}$ is that of Sample A, allowing studies of structures which differ only in profile. The new design produces a high degree of hydrophobicity, with water angles $\theta_{\mathrm{a}}=170 \pm 2^{\circ}$ and $\theta_{\mathrm{r}}=163 \pm 2^{\circ}$. Compared to Sample A, the advancing angle increases by $10^{\circ}$ and the hysteresis decreases by $10^{\circ}$, due to the geometrical reduction of liquid contact.

Nanocones are far less adhesive toward hot water (Figure 3c, red squares) than nanocylinders with the same spacing (Sample A, blue circles) - the surface that previously outperformed all 
larger structures B1-3 (Figure 2d). Within the measurement uncertainty, surface C has nearly no variation of adhesion $\Delta F$ with increasing condensation strength $\Delta T$, in contrast to substrate A where $\Delta F$ increases linearly with $\Delta T$. The conical geometry influences water adhesion in two ways (Figure 3d): as suggested numerically in ref. 32, it impedes condensation at the bottom of the structures, where pillar spacing vanishes; and it provides a Laplace expulsion out of the texture, preventing formation of durable wet patches. These effects conspire to keep the liquid in a full Cassie state, with no increase of adhesion as condensation proceeds, even for isolated microdrops (Figure 3e).

\section{Statistical analysis of the antifogging ability}

We evaluate the promising anti-fogging behaviour of nanocones under the more realistic conditions of dew formation. Samples are placed upside down on a Peltier module and brought to a temperature such that the supersaturation $S$ (ratio between vapour pressure at laboratory temperature and saturated vapour pressure at surface temperature) is kept constant for all experiments, at a value $S=1.7 \pm 0.1$. We observe the breath figures with an inverted microscope over the course of 45 minutes and take one image every $2 \mathrm{~s}$.

Breath figures on nanocylinders (Sample A) markedly differ from those on nanocones (Sample C) (Figure 4a). At short times $(t=20 \mathrm{~s}$ ), both substrates are similarly covered by a large number of microdroplets (radius $\sim 5 \mu \mathrm{m}$ ), but differences become readily apparent after 5 minutes: the cylindrical texture then forms large droplets, while conical structures show only a few of intermediate size and a new generation of microdroplets. This population can be understood from movies (Supplementary Movie 5): growing droplets coalescing with their neighbours irreversibly jump from conical nanotexture, as shown in Figure 4b, with an average size of $9 \mu \mathrm{m}$. As a consequence, the breath figure on Sample C does not evolve with time, while drops keep growing on Sample A. Thus, the volume of water adhered to the solid after 45 minutes falls from $\sim 50 \mathrm{~nL} / \mathrm{mm}^{2}$ on A to only $\sim 5 \mathrm{~nL} / \mathrm{mm}^{2}$ on $\mathrm{C}$. 

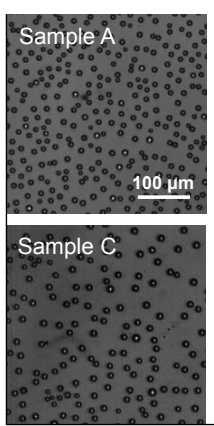

$t=20 \mathrm{~s}$
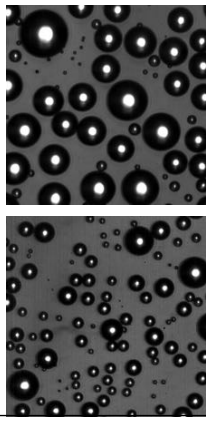

$t=5 \mathrm{~min}$
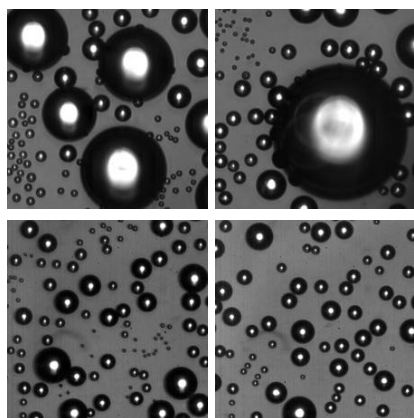

$t=10 \mathrm{~min}$

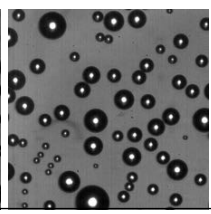

$t=20 \mathrm{~min}$

(a)

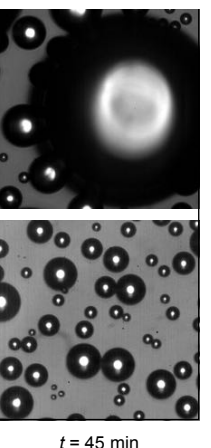

$t=45 \mathrm{~min}$
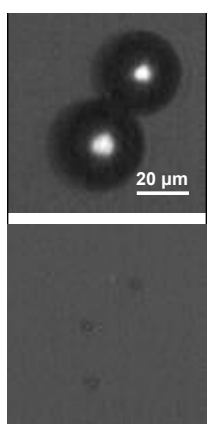

(b)

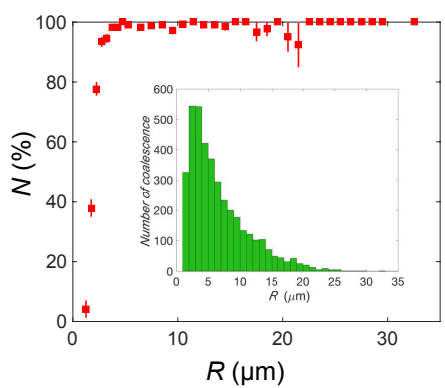

(e)

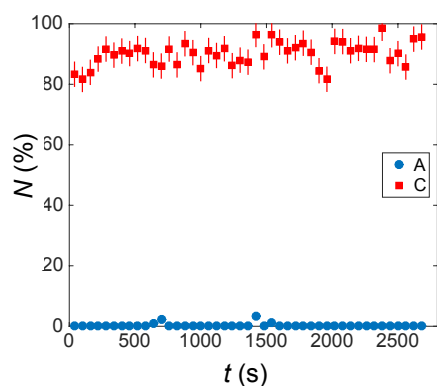

(c)

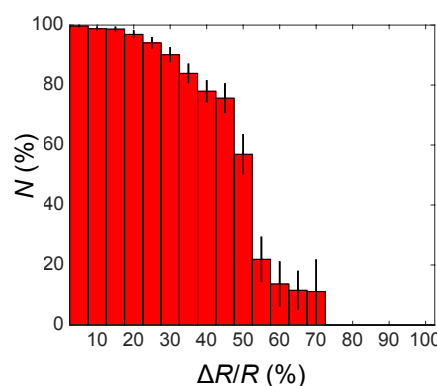

(d)

Figure 4. Condensation of water from a supersaturated atmosphere on nanoscale cones and pillars. (a) Breath figures on samples $\mathrm{A}$ and $\mathrm{C}$ under an optical microscope after 20 seconds, 5 minutes, 10 minutes, 20 minutes and 45 minutes. The scale bar indicates $100 \mu \mathrm{m}$. (b) Snapshots of surface C before (top) and after (bottom) two drops coalesce and jump off. (c) Time evolution of the percentage $N$ of coalescences resulting in droplet jumps for samples A (blue circles) and C (red squares). Each point is obtained by averaging the proportion of jumps over one minute, from a total number of 5500 coalescences for sample $\mathrm{C}$ and 2000 for sample A. (d) Antifogging ability $N$ on material $\mathrm{C}$ as a function of $\Delta R / R$, the relative difference between coalescing drops' radii larger than $4 \mu \mathrm{m} . N$ decreases sharply around $\Delta R / R=0.5$, which corresponds to droplet volumes differing by a factor 10 . (e) Antifogging ability $N$ as a function of $R$, the common radius $(\Delta R / R<0.2)$ of drops coalescing on sample C. $N$ reaches a constant value of $99 \%$ independent of $R$, down to a sharp cutoff value $R_{\min }=1.5 \pm 0.2 \mu \mathrm{m}$. The insert shows the size distribution of drops coalescing on sample $\mathrm{C}$ with $\Delta R / R<0.2$.

We quantify in Figure 4c the antifogging efficiency by measuring the proportion $N$ of drops jumping after coalescence. After observing 7500 coalescences (5500 for the cones, 2000 for the pillars), we plot $N$ versus time; each data point represents an average over one minute, corresponding to typically 150 coalescences on cones, and 50 on pillars. The difference between these numbers arises from the difference in breath figures, which produce more coalescences on cones. For both textures, $N$ is constant in time, but its value dramatically differs. In a cylindrical texture, $N$ is essentially zero (only 5 droplets were observed to take off), while more than $90 \%$ of droplets leave conical features. Jumping droplets have been reported on cicada wings ${ }^{11}$, nanoneedles ${ }^{18}$, Glaco nanobeads ${ }^{22}$ or hierarchical surfaces ${ }^{20-21,33}$ the only case where $N$ was reported ${ }^{33}$ and found to be around $\sim 30 \%$. We show in Supplementary Note 7 that statistics for Glaco under similar conditions gives $N \approx 6 \%$. 
Hence nanocones provide remarkable antifogging abilities: the rare events of coalescence without jump correspond to asymmetric merging, as shown in Figure 4d where the probability of jump $N$ is plotted as a function of the relative difference in radius $\Delta R / R$, for drops larger than $4 \mu \mathrm{m}$. For similar sizes $(\Delta R / R<0.2,2300$ events in a total of 3200 two-body coalescences), we measure $N \approx 99 \%$, producing the impression of extensive departure observed in the accompanying Supplementary Movie 5. However, $N$ falls to $20 \%$ around $\Delta R / R \sim 0.5$, corresponding to a 10 -fold volume difference between droplets. For such marked asymmetries, the smaller drop does not communicate enough momentum to generate takeoff. Error bars and fluctuations also increase, due to the rarity of asymmetric events (100 for $\Delta R / R>0.5$ compared to 3100 for $\Delta R / R<0.5$ in Figure $4 \mathrm{~d}$ ).

The antifogging ability $N$ for similar drops $(\Delta R / R<0.2)$ is finally plotted in Figure $4 \mathrm{e}$ as a function of the average drop size $R$. Antifogging is found to keep its full efficiency $(N \approx 99 \%)$ in a very broad range of radii (from $\sim 3 \mu \mathrm{m}$ to $32 \mu \mathrm{m}$, corresponding to drop volumes differing by a factor 1000), which confirms that the $10 \%$ probability of having no jump in Figure $4 \mathrm{c}$ is mostly due to size contrast between merging drops. The insert shows the coalescence distribution used to perform this statistics. It peaks at small size $(R=2-3 \mu \mathrm{m})$, and the statistics for $R<3 \mu \mathrm{m}$ is based on 870 events. In this range, we observe a modest decrease of $N$ as $R$ decreases, followed by a sharp fall, which defines a cut-off radius $R_{\min }$ for departing. Quantifying $R_{\min }$ as the radius at $N=50 \%$, we measure $R_{\min }=1.5 \pm 0.2 \mu \mathrm{m}$. This quantity can be used as a metrics of water mobility on textures: the smaller $R_{\min }$, the more fog-repellent the material. In the few cases where it was discussed, this distance was found to be typically 10 $\mu \mathrm{m}$, a value attributed to the interactions with the substrate ${ }^{34-35}$. Our value is much smaller, which highlights the extreme mobility of droplets on nanocones, even at a microscale. The value of $R_{\text {min }}$ might be understood by comparing the radius $\ell$ of the contact line to the texture period. On a non-wetting material at small $R$, we have $\ell \approx R\left(\pi-\theta_{\mathrm{a}}\right)$, taking the advancing angle as the relevant one for a growing drop. For $R=R_{\min } \approx 1.5 \mu \mathrm{m}$ and $\theta_{\mathrm{a}} \approx 170 \pi / 180$, we obtain $\ell \approx 250 \mathrm{~nm}$, a value comparable to $90 \mathrm{~nm}$, the largest distance between neighbouring cone tops on sample C. Such droplets (or smaller) may sag between cones, which impedes their mobility.

Hence feature small size was found to improve anti-fogging abilities (as assumed for mosquitoes' eyes ${ }^{10}$ ), an effect maximized by shaping nanofeatures into cones (such as 
observed on cicadae's wings ${ }^{11}$ ). The existence of an optimum of antifogging for dense nanocones could be discussed both by considering intermediate shapes between cylinders and cones, and by diluting nanofeatures. Departing drops also raise interesting questions of fluid dynamics: takeoff speeds can approach $1 \mathrm{~m} / \mathrm{s}$ for micrometric drops, a value much smaller than predicted by a transfer of surface energy in kinetic energy, which remains to be understood. This speed might be sufficient in most natural systems to allow droplets to go with the wind, but what happens if they go back to the substrate remains to be described: at such microscales, air viscosity slows down these drops that can get stuck at impact - except in cases where they meet other condensing drops and participate to their evacuation. More generally, the remarkable water repellency of nanocones arrays, even for microdrops, yields a new kind of platform for manipulating such tiny quantities of cold, or even hot, water.

\section{References}

1. Blossey, R. Self-cleaning surfaces - virtual realities. Nature Materials, 2, 301-306, (2003).

2. Papadopoulos, P., Mammen, L., Deng, X., Vollmer, D. \& Butt, H. J. How superhydrophobicity breaks down. Proceedings of the National Academy of Sciences, 110, 3254-3258, (2013).

3. Cheng, Y.T. \& Rodak, D.E. Is the lotus leaf superhydrophobic? Applied Physics Letters, 86, 144101, (2005).

4. Cheng, Y.T., Rodak, D.E., Angelopoulos, A. \& Gacek, T. Microscopic observations of condensation of water on lotus leaves. Applied Physics Letters, 87, 194112, (2005).

5. Wier, K.A. \& McCarthy, T.J. Condensation on ultrahydrophobic surfaces and its effect on droplet mobility: ultrahydrophobic surfaces are not always water repellent, Langmuir, 22, 2433-2436, (2006).

6. Dorrer, C. \& Rühe, J. Condensation and Wetting Transitions on Microstructured Ultrahydrophobic Surfaces. Langmuir, 23, 3820-3824, (2007).

7. Varanasi, K.K., Hsu, M., Bhate, N., Yang, W. \& Deng, T. Spatial control in the heterogeneous nucleation of water. Applied Physics Letters, 95, 094101, (2009).

8. Liu, Y., Chen, X. \& Xin, J.H. Can superhydrophobic surfaces repel hot water? Journal of Materials Chemistry, 19, 5602-5611, (2009).

9. Narhe, R.D., Gonzalez-Viñas, W. \& Beysens, D. Water condensation on zinc surfaces treated by chemical bath deposition. Applied Surface Science, 256, 4930-4933, (2010).

10. Gao, X.F., Yan, X., Yao, X., Xu, L., Zhang, K., Zhang, J., Yang, B. \& Jiang, L. The dry-style antifogging properties of mosquito compound eyes and artificial analogues prepared by soft lithography. Adv. Mater. 19, 2213-2215, (2007).

11. Wisdom, K.M., Watson, J.A., Qu, X., Liu, F., Watson, G.S. \& Chen, C.-H. Self-cleaning of superhydrophobic surfaces by self-propelled jumping condensate. Proceedings of the National Academy of Sciences, 110, 7992-7997, (2013).

12. Checco, A., Ocko, B. M., Rahman, A., Black, C. T., Tasinkevych, M., Giacomello, A. \& Dietrich, S. Collapse and reversibility of the superhydrophobic state on nanotextured surfaces, Phys. Rev. Lett. 112, 216101, (2014). 
13. Park, K.C., Choi, H.J., Chang, C.H., Cohen, R.E., McKinley, G.H. \& Barbastathis, G. Nanotextured silica surfaces with robust superhydrophobicity and omnidirectional broadband supertransmissivity. ACS Nano, 6, 3789-3799, (2012).

14. Miljkovic, N., Enright, R. \& Wang, E.N. Modeling and optimization of superhydrophobic condensation. J. Heat Trans., 135, 111004, (2013).

15. Miljkovic, N., Enright, R., Nam, Y., Lopez, K., Dou, N., Sack, J. \& Wang, E.N. Jumping-droplet-enhanced condensation on scalable superhydrophobic nanostructured surfaces. Nano Letters, 13, 179-187, (2012).

16. Boreyko, J.B. \& Chen, C.-H. Self-propelled dropwise condensate on superhydrophobic surfaces. Physical Review Letters 103, 184501, (2009).

17. Chen, X., Wu, J., Ma, R., Hua, M., Koratkar, N., Yao, S. \& Wang, Z. Nanograssed micropyramidal architectures for continuous dropwise condensation. Advanced Functional Materials, 21, 4617-4623, (2011).

18. Tian, J., Zhu, J., Guo, H. Y., Li, J., Feng, X. Q. \& Gao, X. Efficient self-propelling of small-scale condensed microdrops by closely packed $\mathrm{ZnO}$ nanoneedles. The Journal of Physical Chemistry Letters, 5, 2084-2088, (2014).

19. Lv, C., Hao, P., Yao, Z., Song, Y., Zhang, X. \& He, F. Condensation and jumping relay of droplets on lotus leaf. Applied Physics Letters, 103, 021601, (2013).

20. Dorrer, C. and Rühe, J. Wetting of silicon nanograss: from superhydrophilic to superhydrophobic surfaces. Advanced Materials, 20, 159-163, (2008).

21. Rykaczewski, K., Paxson, A.T., Anand, S., Chen, X., Wang, Z. \& Varanasi, K.K. Multimode multidrop serial coalescence effects during condensation on hierarchical superhydrophobic surfaces. Langmuir, 29, 881-891, (2013).

22. Lv, C., Hao, P., Yao, Z. \& Niu, F. Departure of condensation droplets on superhydrophobic surfaces. Langmuir, 31, 2414-2420, (2015).

23. Rykaczewski, K., Osborn, W.A., Chinn, J., Walker, M.L., Scott, J.H.J., Jones, W., Hao, C., Yao, S. \& Wang, $\mathrm{Z}$. How nanorough is rough enough to make a surface superhydrophobic during water condensation? Soft Matter, 8, 8786-8794, (2012).

24. Enright, R., Miljkovic, N., Al-Obeidi, A., Thompson, C.V. \& Wang, E.N. Condensation on superhydrophobic surfaces: the role of local energy barriers and structure length scale. Langmuir, 28, 1442414432, (2012).

25. Jiang, Y., Hirvi, J.T., Suvanto, M. \& Pakkanen, T.A. Molecular dynamic simulations of anisotropic wetting and embedding of functionalized polypropylene surfaces. Chem. Phys., 429, 44-50, (2014).

26. Lata Singh, S., Schimmele, L. \& Dietrich, S. Structures of simple liquids in contact with nanosculptured surfaces. Phy. Rev. E, 91, 032405, (2015).

27. Checco, A., Rahman, A. \& Black, C.T. Robust superhydrophobicity in large-area nanostructured surfaces defined by block-copolymer self assembly. Advanced Materials, 26, 886-891, (2014).

28. Reyssat, M. \& Quéré, D. Contact angle hysteresis generated by strong dilute defects. The Journal of Physical Chemistry B, 113, 3906-3909, (2009).

29. Yu, Z.J., Yang, J., Wan, F., Ge, Q., Yang, L.L., Ding, Z.L., Yang, D.Q. \& Isimjan, T.T. How to repel hot water from a superhydrophobic surface? Journal of Materials Chemistry A, 2, 10639-10646, (2014).

30. Khandekar, S. \& Muralidhar, K. Dropwise Condensation on Inclined Textured Surfaces. Springer, 2014.

31. Rykaczewski, K. Microdroplet growth mechanism during water condensation on superhydrophobic surfaces. Langmuir, 28, 7720-7729, (2012).

32. Xu, W., Lan, Z., Peng, B.L., Wen, R.F.\& Ma, X.H. Effect of nanostructures on the nucleus wetting modes during water vapor condensation: from individual groove to nano-array surface. RSC Adv., 6, 7923-7932, (2016). 
33. Rykaczewski, K., Paxson, A.T., Anand, S., Chen, X., Wang, Z. \& Varanasi, K.K. Multimode multidrop serial coalescence effects during condensation on hierarchical superhydrophobic surfaces. Langmuir, 29, 881-891, (2013).

34. Liu, F., Ghigliotti, G., Feng, J. J. \& Chen, C. H. Numerical simulations of self-propelled jumping upon drop coalescence on non-wetting surfaces. J. Fluid Mech., 752, 39-65, (2014).

35. Cavalli, A., Preston, D. J., Tio, E., Martin, D. W., Miljkovic, N., Wang, E. N. \& Bush, J. W. Electrically induced drop detachment and ejection. Phys. Fluids, 28, 022101, (2016).

\section{Acknowledgements}

We thank Direction Générale de l'Armement (DGA) for contributing to the financial support, Rose-Marie Sauvage for her constant interest, and Thales for cofunding this project. Research carried at Brookhaven National Laboratory is supported by the U.S. Department of Energy, Office of Basic Energy Sciences, under Contract No. DE-SC0012704 and used resources of the Center for Functional Nanomaterials, which is a U.S. DOE Office of Science Facility. We finally thank Romain Labbé for help in the design of experiments, and ManeSi-Laure Lee and Brigitte Loiseaux from Thales Research and Technology for many fruitful discussions.

\section{Author contributions}

Ti.M. and D.Q. conceived the project, Ti.M., G.L., Th.M., C.C. and D.Q. designed the project, G.L. and S.X. produced samples B1-3, A.C., A.T. and C.T.B. produced samples A and C, Ti.M. and A.C. performed experiments and analyses, Ti.M., C.C. and D.Q. discussed the models, Ti.M. and D.Q. wrote the manuscript with inputs from all other authors.

\section{Data availability statement}

The datasets generated during current study are available from the corresponding author on reasonable request.

\section{Additional information}

Supplementary information is available in the online version of the paper. Reprints and permissions information is available online at www.nature.com/reprints. Correspondence and requests for materials should be addressed to Ti.M. or D.Q.

\section{Competing financial interests}

The authors declare no competing financial interests. 


\section{Methods}

\section{Model nanotextures}

Sample A: This surface is fabricated by combining block-copolymer self-assembly with anisotropic plasma etching in silicon, which provides large-area $\left(\mathrm{cm}^{2}\right)$ textures with $\sim 10 \mathrm{~nm}$ feature size and long-range order as described in Checco et al. in ref 27. Posts on sample A have a radius $a=15 \mathrm{~nm}$ and a height $h=88 \mathrm{~nm}$, and they are disposed on a rhombus network with side $p=52 \mathrm{~nm}$. The roughness factor $r$ is $r_{\mathrm{A}} \approx 4.5$.

Sample B1, B2 and B3: These textures are square lattices of pillars fabricated by electron-beam lithography and anisotropic plasma etching in silica. These homothetic surfaces have a pillar density of about $10 \%$, an aspect ratio $h / 2 a=3$, and a roughness $r_{\mathrm{B}} \approx 2.2$. The pillar sizes, heights and spacings $a, h, p$ are respectively 100,600 , $560 \mathrm{~nm}$ (surface B1), 150, 900, $840 \mathrm{~nm}$ (B2), and 200, 1200, $1120 \mathrm{~nm}$ (B3).

Sample C: Nanocones are fabricated by using the exact same method as for sample A, and the only difference comes during the etching step. To obtain conical shape, etching is made more isotropic as described in Checco et $a l$. in ref 27. Cones on sample $\mathrm{C}$ have a height $h=115 \mathrm{~nm}$, and a texture's spacing (or base diameter) $p=52 \mathrm{~nm}$, as for Sample A. Cones have a roughness factor $r_{\mathrm{C}} \approx 4.2$, close to that of $\mathrm{A}$.

Chemical vapour deposition is made by activating the surface in a plasma cleaner during 45 seconds. The activated surface is enclosed in a Petri dish close to a plastic well containing typically $\sim 20 \mu \mathrm{L}$ of $1 \mathrm{H}, 1 \mathrm{H}, 2 \mathrm{H}, 2 \mathrm{H}-$ perfluorodecyltrichlorosilane and desiccants to avoid reaction between the silane and water contained in the air. The silane used is referenced as L16584.03 in VWR.

\section{Condensation induced adhesion}

Immersing the syringe and the connecting tube in a thermostated liquid controls the drops' temperature. The thermostated water is contained in a cylindrical copper pipe closed with a brass plate where the needle is attached. We control the temperature via the voltage applied to a silicon heater mat (referenced as 245-534 on RS Components) glued to the copper container and precisely monitored with a temperature sensor.

\section{Condensation observations}

We place the substrate on a Peltier module (referenced as 693-7043 on RS Components) whose hot side is cooled with a heat sink coupled to a fan (commonly used for CPU cooling). The samples are brought to a temperature such that the supersaturation $S$ (ratio between vapour pressure at laboratory temperature and saturated vapour pressure at surface temperature) is kept constant for all experiments at a value $S=1.7 \pm 0.1$. The samples are mounted upside down on the Peltier module's underside, so that any droplets jumping from the surface do not return. We observe the breath figures during 45 minutes using an inverted microscope (Amscope IN300-FL) connected to a high-resolution video-camera (Photron Fastcam SA3). 